\title{
Perception about the use of monitoring systems in civil construction structures
}

\author{
Percepción sobre el uso de Sistemas de monitoreo en estructuras de construcción civil
}

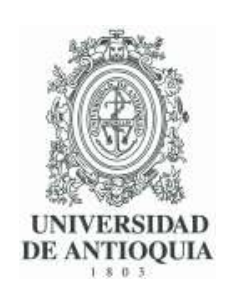

\author{
Claudio Alcides Jacoski ${ }^{*}$ Larissa Saugo $^{2}$ \\ ${ }_{1}^{1}$ Postgraduate Program in Technology and Innovation Management, Community University of the Region of Chapecó - Unochapecó. Rua \\ Servidão Anjo da Guarda, 295-D, C. P. 89809-900 Chapecó, Brazil \\ ${ }^{2}$ Program of Exact and Environmental Sciences, Community University of the Region of Chapecó - Unochapecó. Rua Servidão Anjo da \\ Guarda, 295-D. C. P. 89809-900. Chapecó, Brazil
}

ARTICLE INFO:

Received: May 07, 2018

Accepted: December 13, 2018

\section{AVAILABLE ONLINE:}

December 13, 2018

\section{KEYWORDS:}

Sensors, monitoring, reinforced concrete structure, buildings

Sensors, monitoreo, estructura de hormigón armado, edificios
ABSTRACT: In recent years, concrete structures have started to show increased amounts of degradation or damage. This phenomenon is generally caused by the growth of these constructions, particularly with regard to slenderness. In the quest for new strategies to address this problem and to ensure the safety of structures and their occupants, tools for damage detection and timely analysis distributed within buildings have been developed. These tools are referred to as "Structural Health Monitoring" sensors. It is known that these systems still have high costs, thus this study aims to assess their value through future projections of their costs and utility. This monitoring method, although not widespread, mainly aims to maintain the integrity of major projects; it is not currently focused in small sized works, with the exception of a few structures of historical interest. Therefore, this study proposes the analysis of structural monitoring systems in civil construction, through means of a survey and interviews with experts. The study is aimed at investigating current technologies, planning for implementation, and identifying costs and feasibility of structural monitoring in the future. In conclusion, it was possible to expose that deformation and displacement sensors, along with optical and electrical technologies are the most commonly used types of sensors. Furthermore, it was found that monitoring systems are only feasible for large structures and the estimated time for use in medium-sized buildings is 15 years or more. However, it can be noted that the cost is currently very high, but will likely be reduced in the future.

RESUMEN: En los últimos años, las estructuras de hormigón han comenzado a mostrar una mayor cantidad de degradación o daños. Este fenómeno es causado generalmente por el crecimiento de estas construcciones, especialmente con respecto a la esbeltez. En la búsqueda de nuevas estrategias para hacer frente a este problema y para garantizar la seguridad de las estructuras y de sus ocupantes, se han desarrollado herramientas para la detección y el análisis de daños distribuidos dentro de los edificios. Estas herramientas se conocen como sensores de monitoreo de salud estructural (SHM). Se sabe que estos sistemas todavía tienen altos costos, por lo que este estudio tiene como objetivo evaluar su valor a través de las proyecciones futuras de sus costos y utilidad. Este método de monitorización, aunque no se ha generalizado, principalmente tiene como objetivo mantener la integridad de los grandes proyectos; no se centra actualmente en el seguimiento de las obras pequeñas y medianas empresas, con la excepción de unas pocas estructuras de interés histórico. Por lo tanto, este estudio propone la visualización y análisis de sistemas de monitoreo estructurales de la construcción civil, a través de medios de una encuesta y entrevistas con expertos. El objetivo del estudio es investigar tecnologías y aplicaciones actuales, la planificación de la implementación, y la identificación de costos y viabilidad de la supervisión estructural en el futuro.

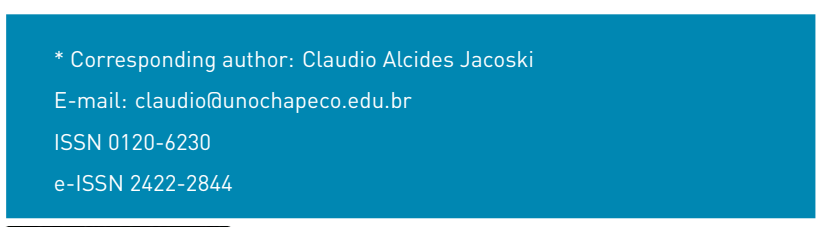


En conclusión, fue posible exponer que la deformación y desplazamiento sensores, junto con las tecnologías ópticas y eléctricas son los tipos más comúnmente utilizados de sensores. Además, se ha descubierto que los sistemas de vigilancia son solamente factibles para grandes estructuras y el tiempo estimado para su uso en edificios de tamaño medio es de 15 años o más. Sin embargo, se puede señalar que el coste es actualmente muy alto, pero es probable que se reduzca en el futuro.

\section{Introduction}

Currently, reinforced concrete is the primary material used in civil structures. However, in recent years various issues have developed from the use of inadequate and outdated construction and drafting techniques, increased structural requirements, and increased complexity of shapes and architectural aspects. Nowadays, constructions, both large and small, have been receiving increased attention in Brazil as they have been subjected to defects, such as fissures, that can result in considerable damage. Such damage typically requires repairs that subsequently need monitoring, maintenance, and restoration.

Throughout the world, various structures have been monitored and observed by [1-3]. Though this, large building, not small buildings, have been the target of interest.

Most accidents occur in buildings because the owners and professionals ignore the degradation and malfunctioning of such structures [4]. Thus, monitoring these buildings would greatly contribute to their safety, warning the owners of the existence of precarious conditions and providing preventive solutions. Furthermore, this could improve the accuracy and practicality of future projects.

Structural monitoring systems are essentially composed of sensors and acquisition systems and use diagnostics (data processing and analysis) to compile interpretations [4].

These sensor assemblies may include electric, optical, mechanical, photometric, photogrammetric, or geodetic technologies. The networks are used to associate more than one feature into a sole system [5]. Moreover, the sensors available in the market for civil constructions include deformation, temperature, displacement, strain, load, humidity, pore pressure, carbonation front, and corrosion potential sensors.

The existence of these systems allows for the detection of damage within structures, improving performance and quality by providing real and reliable information with regard to the structures. Poor implementation can cause problems with severe consequences including economic, cultural, and social impacts, depending on the function of the structure.

As mentioned by [6], systems can have a major impact on analyzing safety through identification of damage and durability. Their implementation could bring about a new way of assessing and monitoring structures of civil construction, focusing on human health, identifying the need for intervention when appropriate, diagnosing the causes of undesirable conditions, and taking preventative measures.

Another key component of effective monitoring is the detection of structural behavior changes caused by natural disasters, such as earthquakes, hurricanes, tornadoes fires, and floods (which apply specifically to Brazil). Post-occurrence earthquake applications were implemented in the Punggol project in Singapore [1, 7].

However, installing monitoring systems is expensive, which is probably the reason for their absence in smaller buildings, despite the fact that they have been recent problem targets. Perhaps these deficiencies can be avoided with improved, detailed, precautious design that calls for quality materials with durability compatible with the previewed timeframe, so as to avoid the need for reworking. Thus, the monitoring systems would only have preventive functions.

This study was intended to estimate the feasibility of monitoring systems across large, medium, and small structures. Small building constructions were specifically investigated for their limitations pertaining to structural monitoring systems.

\section{Methodological procedures}

For the completion of this study, data was collected through a survey, following the principles of the Delphi method. According to [8], the Delphi method employs a synthesis of expert opinions regarding new developments and future trends for which there is a lack of existing data.

The survey was based on existing doubts related to structural monitoring, with its purpose being to identify a trend regarding future projections. The survey was administered along with some background information on the study, sent via e-mail to experts from around the world.

The survey was sent to 15 experts, all of which had conducted research on the application of structural monitoring, including two with civil engineering degrees, three with master's in civil engineering, and ten with 
Table 1 Average choice on preference regarding the use of sensors

\begin{tabular}{lccccccccc}
\hline SENSOR / EXPERTS & [1] & [2] & [3] & [4] & [5] & [6] & [7] & [8] & AVERAGE \\
\hline Loads & 3 & 3 & 3 & 4 & 3 & 2 & 4 & 3 & 3.125 \\
Deformation & 2 & 1 & 1 & 1 & 2 & 1 & 2 & 1 & 1.375 \\
Displacement & 1 & 2 & 2 & 3 & 1 & 3 & 1 & 2 & 1.875 \\
Carbonation Front & 5 & 9 & 8 & 9 & 9 & 6 & 8 & 7 & 7.625 \\
Pore Pressure & 8 & 8 & 7 & 7 & 8 & 7 & 7 & 8 & 7.5 \\
Corrosion Potential & 7 & 7 & 5 & 8 & 7 & 9 & 9 & 9 & 7.625 \\
Temperature & 4 & 4 & 4 & 2 & 5 & 4 & 3 & 4 & 3.75 \\
Voltage & 9 & 6 & 6 & 5 & 4 & 5 & 6 & 5 & 5.75 \\
Humidity & 6 & 5 & 9 & 6 & 6 & 8 & 5 & 6 & 6.375 \\
\hline
\end{tabular}

doctorates in civil engineering. The profiles of these experts were found on the internet, either through the university websites where they teach, or through their official professional profiles.

As opposed to completing the survey, one expert contributed by providing comments through a recorded interview via the Skype application. This occurred on a certain time and date.

The survey was developed through inquiries referencing bibliographies, and published case studies. Ten questions were created, six of them being objective and four of them being discussion-based.

The objective questions were related to general aspects of the monitoring system, and the subjective questions dealt with the research conducted by the experts. Additional information such as the filling date (which was automatic), name (optional), training (required), and place/country of filling (required) was requested.

About the research, it is noted that only a few experts returned with the answers. Seven experts answered the published survey and one professor provided feedback orally via Skype, totaling responses from eight experts or $53.33 \%$ of those administered the survey. From the sample one had a degree in civil engineering, two were Master of Civil Engineering, five were Doctor of Civil Engineering.

The interview was recorded, analyzed, and enclosed along with the responses from the published surveys. The collected data was considered question-by-question, targeting each issue separately.

For the research, a form developed for semi-structured research was used, so that besides the items pointed out in the questionnaire, it was possible to elucidate questions using the recording of the interview.

\section{Study on structural monitoring systems}

\subsection{Use of structural monitoring sensors}

In the field, the use of sensors for structural monitoring cannot be implemented since the sensors are not available in sufficiently large quantities for the commercialization of this technology. Among the technologies used by companies, other types of sensors seemed to be used more often in comparison. In the literature, there are several other types of sensors used, as shown [9-13].

The following types of sensors comprise the possible alternatives: deformation, temperature, displacement, voltage, loads, humidity, pore pressure, carbonation front, and corrosion potential sensors. It was predicted that deformation and displacement sensors are the most used as they are available to all commercial companies and have a higher reported use. The eight experts ranked their choices from most used to least used, and with this information, averages were obtained (Table 1).

With this, it can be observed that none of the choices were unanimous. With the results of the averages, it was possible to sort the sensors based on usage (Table 2).

Table 2 Sensor Rate of use

\begin{tabular}{ccc}
\hline CLASSIFICATION & SENSOR & AVERAGE OF EXPERTS \\
\hline 1 & Deformation & 1.375 \\
2 & Displacement & 1.875 \\
3 & Loads & 3.125 \\
4 & Temperature & 3.75 \\
5 & Voltage & 5.75 \\
6 & Humidity & 6.375 \\
7 & Pore Pressure & 7.5 \\
8 & Carbonation Front & 7.625 \\
9 & Corrosion Potential & 7.625 \\
\hline
\end{tabular}

It is important to note that the deformation and displacement sensors were found to be the most commonly used. This was probably due to the fact that 
deformation sensors are responsible for assessing the tensions in structures in a direct way, and displacement sensors measure the overall resistance, resulting in monitoring of the most important factors.

The third most commonly used type of sensor was load sensor, which is also useful for estimating the intensity and the load within the structures. As for temperature sensors, they were found to be frequently used together with other sensors to read precise values relating to temperature fluctuations, justifying shrinkage and expansion phenomena without any structural details. The remainder of the sensors mentioned were found only to be used to evaluate factors related to the durability of the structures, so they are provisional and less commonly used in civil construction.

\subsection{Technologies used in monitoring systems with relation to the cost-benefit}

Currently, there are many types of technologies available for use in structural monitoring. Amongst these are mechanical, optical, electrical, photometric, and geodetic sensors. Electrical and optical sensors were expected to demonstrate the best cost-benefit as they are the types appearing in most case studies and established applications. The most commonly used technologies according to the experts consulted in this study are shown in Figure 1.

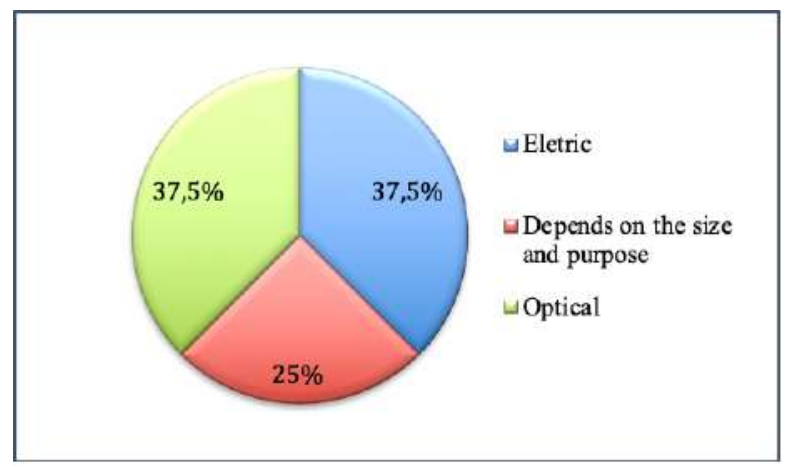

Figure 1 Technologies x Cost-Benefit

The most used technologies were found to be based on optical and electrical networks. It was found that $37.5 \%$ of the experts believe that the best technology is optical, while the same percentage of experts believe that electrical networks are estimated to provide the best cost-benefit. The other $25 \%$ of surveyed experts reported that the most cost-effective technology depends on the network size as well as the goal.

Therefore, experts' choices were mainly dependent on the characteristics of each technology. The electrical and optical networks were perceived similarly. Optical fibers can carry more data and can use up to hundreds of sensors within a single network; however, they are more expensive than other types. Electric sensors, on the other hand, are readily feasible for smaller networks, but have a reduced capacity to carry data over time. Therefore, the optimal technology depends largely on the size of the structure, quantity, and time of the application. The other types of technologies are more common, specific, and rarely used in civil engineering.

\subsection{Buildings where use of SHM is feasible}

Existing references shed light on the elevated costs of structural monitoring systems. Thus, one survey question was dedicated to whether or not such systems would be viable for buildings of all sizes, or if they are only practical in large buildings with specific characteristics, where the values can be stretched or even completely preserved.

The available answers to this question were: the systems are reasonable in all cases, only for large buildings, only for medium and large buildings, or they are not viable. The eight experts' responses are shown in Figure 2.

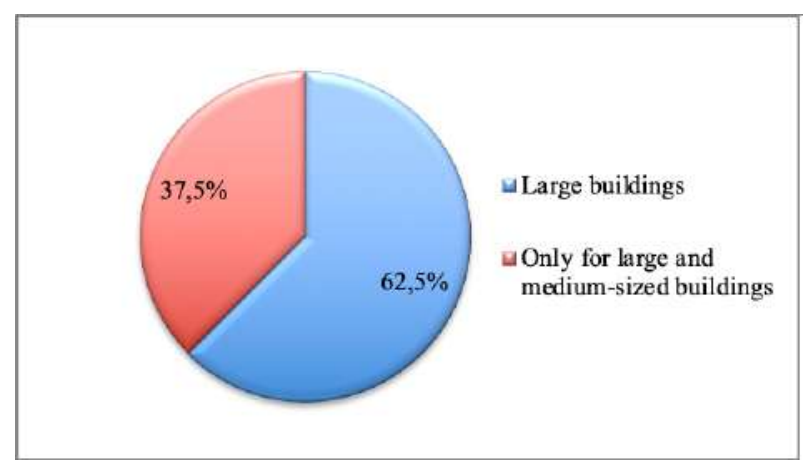

Figure 2 Operating Feasibility of SHM in buildings

As noted in the chart, all experts believed it to be feasible to use monitoring systems in large buildings, 37.5\% also targeted application in medium-sized structures. None, however, envisioned implementing monitoring systems in small buildings.

This shows that the systems are very expensive and are infrequently used in medium buildings and not used at all in small buildings. This results from the fact that much of the instrumentation must be imported, and the location where the systems are to be used is usually difficult to access, requiring skilled labor for installation, maintenance, and data filtering. Also, the sensors and networks are usually built into the structure and therefore cannot be retrieved. Due to these aspects, monitoring needs to be justified, reasonable, and calculated. 
For small and medium sized buildings, the costs of implementing structural monitoring systems usually has a value above expectations of a construction of this size, which nullifying their purpose. Thus, these systems are much more suitable for large buildings, which often have a structure of significant size and justify the investiment.

\subsection{Main types of structures to be monitored}

Through published studies on structural monitoring systems, it was estimated that the possible types of structures to be monitored include large constructions, bridges, viaducts, dams, historic buildings, mid-size buildings, small buildings, and tunnels.

It was believed that the structures with the greatest potential for monitoring would be larger and at higher risk of damage. Thus, the experts rated various structures as what they believed to be the most monitored structures to the least monitored, and through this, averages were obtained (Table 3).

The results show that the experts unanimously voted for the seventh and eighth place structures, which further confirms the lack of interest in monitoring small and medium buildings. Thus, with the average results, it was possible to classify the buildings according to the monitoring system usage (Table 4).

The results verify the consensus the increased monitoring of structures with higher risk levels, such as bridges and dams, which in case of malfunctions, may hinder entire regions or result in massive damage.

In Brazil, sensors still have limited use that are restricted to a few dams; outside of Brazil, the use of monitoring systems is much more extensive, and includes a significant number of large structures, in addition to some slender buildings, where the monitoring is performed continuously. In the meantime, it is important for monitoring to be considered in building designs and put to use in places where there is greatest need. As previously stated, such systems are likely not feasible for the majority of small buildings.

\subsection{Motivation for structural monitoring}

As listed above, the structural monitoring should be planned and justified, and may be motivated by several factors. The possible options in the survey included existing conditions (defects), structure care, accident prevention, educational means (scholastic foundation, calculation program creation, etc.), and proof of numerical methods in addition to the option of listing another motivation. It was expected that the factors relating to structural care and accident prevention would be the most popular, and this was confirmed by the survey responses (Figure 3).

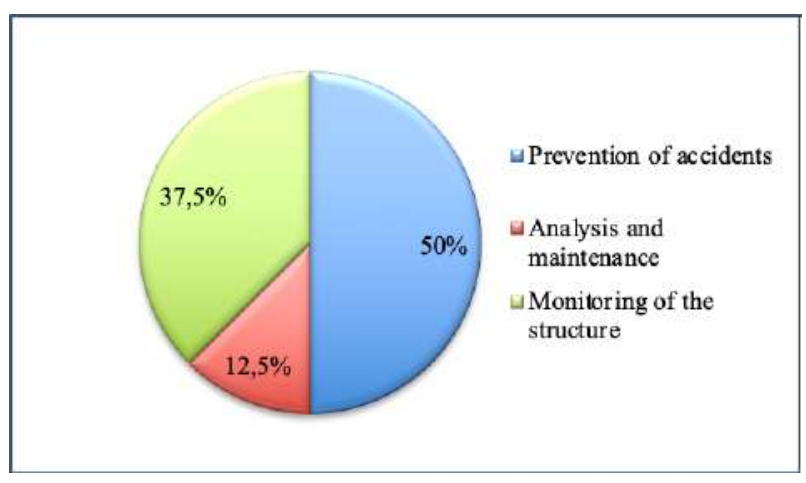

Figure 3 Motivations for structural monitoring

Fifty percent of the experts reported that preventing accidents was most important, $37.5 \%$ selected structure care, which is directly related to accident prevention, and one researcher $(12.5 \%)$ opted numerical methods and maintaining the structure to be the most important. In general, the experts focused on taking care of structures, thus preventing accidents.

It is important to note that each monitoring system has its specific purposes, and like a structural draft, each building has its own particularities, dimensions, and relations. Depending on the needs and time of application, each system requires its own unique configuration.

Theoretically, all types could be combined into a single monitor, but this would affect the quality. Even to calibrate numerical methods, for educational means and for monitoring the conditions, the monitor takes care of the structure and prevents accidents.

Moreover, with respect to monitoring pre-existing conditions, these systems can be used to assess the severity and trajectory of the damage, otherwise requiring a professional diagnosis. If a condition is detected after system installation, a survey must be performed, and decision made on the inspection. Thus, all items have specific value, although the majority converge to accident prevention and structure care.

\subsection{Projections for the use of monitoring systems in medium-sized buildings}

With a better understanding of the monitoring systems and their implications, it is possible to list structure care as being viable and necessary for structures and large buildings, especially works of art. These systems ensure 
Table 3 Classification of structures to be monitored

\begin{tabular}{lccccccccc}
\hline SENSOR / EXPERTS & [1] & [2] & [3] & [4] & [5] & [6] & [7] & [8] & AVERAGE \\
\hline Dams & 2 & 1 & 1 & 1 & 4 & 2 & 2 & 2 & 1.875 \\
Midsize Buildings & 7 & 7 & 7 & 7 & 7 & 7 & 7 & 7 & 7 \\
Small Buildings & 8 & 8 & 8 & 8 & 8 & 8 & 8 & 8 & 8 \\
Historic Buildings & 6 & 6 & 5 & 6 & 6 & 5 & 6 & 6 & 5.75 \\
Large Constructions & 5 & 5 & 6 & 4 & 5 & 6 & 5 & 4 & 5 \\
Bridges & 1 & 2 & 3 & 2 & 1 & 1 & 1 & 1 & 1.5 \\
Tunnel & 3 & 4 & 2 & 3 & 2 & 3 & 4 & 5 & 3.25 \\
Viaducts & 4 & 3 & 4 & 5 & 3 & 4 & 3 & 3 & 3.625 \\
\hline
\end{tabular}

Table 4 Classification of structures to be monitored

\begin{tabular}{ccc}
\hline CLASSIFICATION & STRUCTURE & AVERAGE OF EXPERTS \\
\hline 1 & Bridges & 1.5 \\
2 & Dams & 1.875 \\
3 & Tunnels & 3.25 \\
4 & Viaducts & 3.625 \\
5 & Large Constructions & 5 \\
6 & Historic Buildings & 5.75 \\
7 & Midsize Buildings & 7 \\
8 & Small buildings & 8 \\
\hline
\end{tabular}

higher safety and help prevent accidents and that occur from a lack of prevention and planning measures.

However, it is known that these systems are very expensive in medium and small buildings, thus making their use unpopular. A question was included in the survey regarding the amount of time projected for the systems to be viable in medium-sized buildings. The options included: 5 years, 10 years, 15 years, 20 years, or over 20 years. The results are depicted in Figure 4.

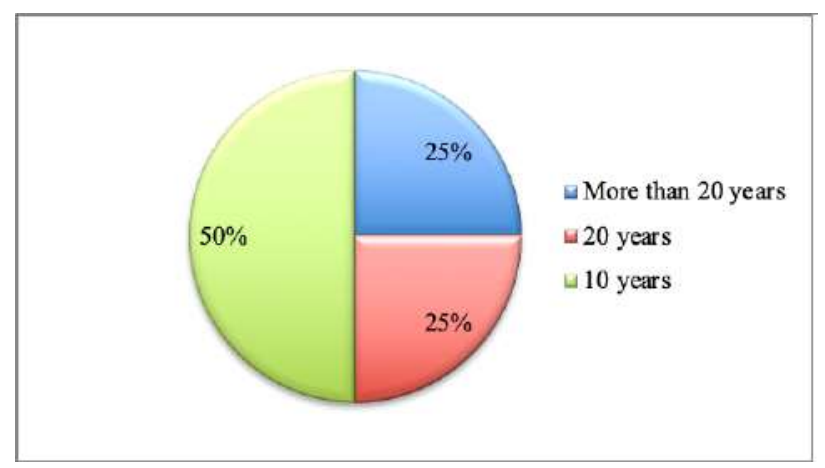

Figure $\mathbf{4}$ Time for the practicality of SHM in medium-sized buildings

Despite the use of structural monitoring systems in large buildings being readily available, as explained previously, the future projection for work in smaller buildings does not seem optimistic. Fifty percent of the researchers believed that practical use would not be implemented prior to 20 or more years. The other $50 \%$ predicted that the monitoring systems would be implemented in medium-sized buildings within 10 years, amounting to an average projection of 15 years or more.

Therefore, this projection is still very distant and unreliable since there is much uncertainty regarding the expansion of structural monitoring. This expansion depends largely on logistics, conditions, reduced costs of technology, and professional employees associated with the systems.

Moreover, especially in Brazil, there is not an emphasis in preventing damage to structures, especially by drafters and companies. Thus, it is necessary to first change the culture, create laws that incentivize structure care, especially those at high risk, and finally, pursuing these systems as a potential tool to provide project verification, and to increase the life of buildings and their safety.

\subsection{Enterprises to obtain the sensors and acquisition systems}

It was believed that monitoring systems could be obtained through several companies and that they would be affordable, and have good quality. However, there are not many companies that sell sensors and data acquisition systems, especially in Brazil since the technology is not overly used.

As for the eight experts who responded to the survey and previously researched the area, the companies through which they obtained their systems included HBM, FiberSensing, Gavea Sensors, and Smartec. The connector [6] developed these sensors and acquisition systems along with the research team in the Telecommunications Institute of Aveiro - Portugal.

In general, the attainment of systems and sensors depends largely on logistics, for there are specific providers for certain lines of work. In Brazil, there are still a few companies that provide these types of products, the majority being imported from companies such as Kyowa (electric technologies) and HBM (optical technologies), for example. 


\subsection{Quantity of sensors and acquisition 4. Conclusions systems}

The experts that filled out the survey were asked to specify the number of sensors and acquisition systems they used in their research. However, in response, they only provided the numbers already published in their research.

Generally, the equipment quantity proved to be highly variable, as each application was unique with a different structure. Thus, estimating a set number would be impractical without checking requests and performing calculations, since designing a monitoring system resembles the draft of a structural design.

\subsection{Physical area covered by the sensors}

It has been noted that each sensor covers a given area, however, the responses from the experts were inconclusive.

This is basically because each monitor is unique and must be measured for each case.

\subsection{Cost of sensors and acquisition systems}

One intention of the study was to obtain the detailed costs and purchase dates or negotiations to trace the estimated future projection for use in assessing the feasibility of structural monitoring systems for smaller structures.

Only two experts had actual values from the monitoring systems. One of them was developed by [6] and the Department of Physics, at the University of Aveiro, with a cost of $€ 50$ per sensor and $€ 300$ for the acquisition system. Another cost was listed was from the Alto do Ipiranga Station [5], where they spent $\$ 40,000$ on the instrumentation, which comprised 10 sensors and an acquisition system.

Thus, even if the systems are from different dates and currencies, it can be said that there are very large disparities between the systems marketed by companies in the industry and the one developed by the University of Aveiro, which proves that there is a tendency to reduce the costs.

In general, cost trends cannot be reliably predicted.

However, it is thought that the cost will be reduced over time, depending on several factors, such as manufacturers, foreign currency (dollar and euro), logistics, being subject to negotiation, and the spread of technology. Thus, each system has different prices, but at the moment, they are expensive.
It can be observed that the experts are vastly knowledgeable on structural monitoring systems, which provides us the opportunity to see several authors' monitoring applications in buildings having different characteristics and sizes. However, their responses were not always consistent with each other, mainly because each monitoring was specific and unique.

It was not possible to precisely estimate the cost of the monitoring systems, and with that, tracing their future projections. There were only two clearly defined values, proving that despite the current costs being too high, the trend was found to decrease, mainly due to the differences between the commercial and production costs of the sensors and acquisition systems. Thus, it is known that the costs are excessively high for smaller structures, but that regardless, are worth the money for structures of great value and high risk, such as for works of art.

Generally, the costs of monitoring systems are currently decreasing due to increased access to technology; however, there is still in need to solve logistical issues that relate mainly to labor, such as the lack of specialization that results in high costs.

Therefore, small- and medium-sized structures do not arouse the interest of specialists as potential candidates for monitoring systems. Their interest lies instead in larger structures and works of high risk, which is primarily driven by the high costs that often exceed the value of the buildings, thereby inhibiting their use.

Furthermore, it is not possible to predict the exact numbers of sensors or data acquisition systems, as well as the physical area they will be able to cover because, as mentioned above, each monitoring system has its own characteristics, just like structural design.

When it comes to sensors, they are used in accordance with the needs and characteristics of the buildings and constructions. Generally, in civil works, strain, displacement, and load sensors are the most common type of sensors used and can monitor the greatest number of parameters within these structures. Still, it is necessary to use temperature sensors in conjunction with others, since the weather and temperature can affect structures with common phenomena, like expansion and contraction. It must be confirmed that thermally evoked changes in the structures are not harmful to the buildings.

Even though the companies that market the required monitoring instrumentation are limited, larger ones, such as HBM, Smartec and Kyowa exist. 
Optical and electrical sensors are most common types of sensors used when it comes to technologies, as cited by many sources. Optical, being more expensive, is more useful for larger networks because it can transmit data to several hundred sensors. Electrical sensors are typically more economical, but have a reduced capacity for transmitting data, and consequently, cannot be linked to as many sensors.

The projections on system usage concluded that their use is feasible in large buildings; however, in medium-sized buildings, the prospects are not optimistic; $50 \%$ of the experts believe that they will be in use in 20 years or more, and the other $50 \%$ estimate that they will be in use in 10 years, for an average of a projected 15 years or more until full implementation.

However, it is believed that structural monitoring systems that use sensors will not become feasible for small buildings, as they may be monitored in a professional, simple, and inexpensive way, since access is facilitated and thus, it is not necessary to design prevention and study the installation of systems.

Currently, in medium-sized buildings, with the cheapening of technology and automation of prevention processes, it is expected that the culture of drafters and customers will adapt, and that monitoring systems will be used. Thus, long-term savings can be achieved through reducing unnecessary maintenance expenses, protecting the occupants and the structure, reducing the number of conditions, and increasing the safety of buildings. As the experts mentioned, the main motivating aspects of monitoring are to prevent accidents and take care of the structure.

Overall, the objectives were partially achieved, and the results are relevant for understanding the implementation of structural monitoring and its features. The limited success was largely based on the fact that not many experts participated in the survey and were often not willing to openly share their knowledge. Another failure factor had to do with to the survey, which could have been more broad and explanatory, or even entirely colloquial.

Finally, it was possible to conclude that the usage of structural monitoring systems by means of sensors is feasible for large structures and buildings, and that it will take time for such systems to be used in smaller buildings. However, what should be stressed is that draftsmen should pay greater attention to the care and prevention of dangerous conditions and structural damage, in order to ensure safety and reliability within civil construction.

\section{References}

[1] B. Glisic, L. J. Ming, N. C. Tat, D. Inaudi, and Y. T. Yew, “Large-scale lifespan monitoring of high-rise buildings using long-gauge fiber optic sensors," in 3rd International Conference on Structural Health Monitoring of Intelligent Infrastructure, Columbia, Canadá, 2007, pp. $1-7$.

[2] A. Arêde, A. Costa, C. Costa, P. M. Costa, and C. Barbosa, "Monitorização do comportamento estrutural de uma ponte nova em arcos de alvenaria de pedra," in 06 Congresso Nacional Mecânica Experimental, Zadar, Croatia, 2004, pp. 184-193.

[3] R. G. de Andrade, "Monitoramento de curta duração de uma ponte curva em concreto armado: um estudo de caso." M.S. thesis, Universidade Politécnica de São Paulo, São Paulo, Brasil, 2012.

[4] A. BRASIL, Monitoreo en la Construcción Civil. Asociación Latinoamericana de Control de Calidad, Patología y Recuperación de la Construcción - ALCONPAT Int, 2013.

[5] W. S. Assis, “Sistemas computacionais de apoio à monitoração de estruturas de engenharia civil," PhD Thesis, Curso de Engenharia Civil, Universidade Politécnica de São Paulo, São Paulo, Brasil, 2007.

[6] P. F. C. Antunes, "Sensores ópticos para monitorização dinâmica de estruturas," Ph.D thesis, Departamento de Física, Universidade de Aveiro, Aveiro, Portugal, 2011.

[7] B. Glišić, D. Inaudi, J. Lau, Y. Mok, and C. Ng, “Long-term monitoring of high-rise buildings using long-gage fiber optic sensors," in 7th International conference on multi-purpose high-rise towers and tall buildings, Dubai, 2005, pp. 1-13.

[8] T. J. Gordon, "The real-time delphi method: excerpt from futures research methodology," Patent, 2008.

[9] S. N. Yu, J. H. Jang, and C. S. Han, "Auto inspection system using a mobile robot for detecting concrete cracks in a tunnel," Automation in Construction, vol. 16, no. 3, pp. 255-261, May. 2007.

[10] M. Sarker, T. Ali, A. Abdelfatah, S. Yehia, and A. Elaksher, “A cost-effective method for crack detection and measurement on concrete surface," ISPRS - International Archives of the Photogrammetry, Remote Sensing and Spatial Information Sciences, vol. XLII-2/W8, pp. 237-241, Nov. 2017.

[11] X. Zhong, X. Peng, S. Yan, M. Shen, and Y. Zhai, “Assessment of the feasibility of detecting concrete cracks in images acquired by unmanned aerial vehicles," Automation in Construction, vol. 89, pp. 49-57, May. 2018.

[12] A. Mohan and S. Poobal, "Crack detection using image processing: A critical review and analysis," Alexandria Engineering Journal, vol. 57, no. 2, pp. 787-798, Jun. 2018.

[13] Y. Zhang, T. Planès, E. Larose, and A. Obermann, “Diffuse ultrasound monitoring of stress and damage development on large scale concrete structures," The Journal of the Acoustical Society of America, vol. 141, no. 5, pp. 3831-3831, 2017. 